\title{
chemosensors
}

ISSN 2227-9040

www.mdpi.com/journal/chemosensors

Article

\section{Development of an Electrochemical Sensor for NADH Determination Based on a Caffeic Acid Redox Mediator Supported on Carbon Black}

\section{Chiara Zanardi 1,*, Erika Ferrari ${ }^{1}$, Laura Pigani ${ }^{1}$, Fabiana Arduini ${ }^{2}$ and Renato Seeber ${ }^{1,3}$}

1 Department of Chemical and Geological Sciences, University of Modena and Reggio Emilia, via G. Campi, 41125 Modena, Italy; E-Mails: erika.ferrari@unimore.it (E.F.); laura.pigani@unimore.it (L.P.); renato.seeber@unimore.it (R.S.)

2 Department of Chemical Sciences and Technologies, University of Tor Vergata, via della Ricerca Scientifica, 00133 Roma, Italy; E-Mail: fabiana.arduini@uniroma2.it

3 Institute of Organic Synthesis and Photoreactivity (ISOF) - National Research Council of Italy (CNR), via Gobetti 101, 40129 Bologna, Italy

* Author to whom correspondence should be addressed; E-Mail: chiara.zanardi@ unimore.it; Tel.: +39-059-205-5033; Fax: +39-059-373-543.

Academic Editors: Paolo Ugo and Michael Ongaro

Received: 27 February 2015 / Accepted: 1 April 2015 / Published: 13 April 2015

\begin{abstract}
Screen-printed electrode (SPE) modified with carbon black nanoparticles (CB) has been tested as a new platform for the stable deposition of caffeic acid (CFA) on the electrode surface. The electrochemical performance from varying the amount of CFA/CB composite has been tested with respect to NADH determination. The electrocatalytic activity of CFA/CB has also been compared with that of SPEs modified by a single component of the coating, i.e., either CFA or CB. Finally, glycerol dehydrogenase, a typical NADH-dependent enzyme, was deposited on the CFA/CB coating in order to test the applicability of the sensor in glycerol determination.
\end{abstract}

Keywords: screen-printed electrodes; amperometric sensor; enzymatic biosensors; NADH; caffeic acid; carbon black; glycerol dehydrogenase; glycerol determination 


\section{Introduction}

Many chemical parameters that identify the quality of foodstuffs can be evaluated by biosensors, containing a suitable enzyme as the recognition element. A particularly high portion of the enzymes used belongs to the family of hydrogenases, which involves nicotinamide adenine dinucleotide $\left(\mathrm{NAD}^{+}\right)$ as the co-factor. For this reason, the development of efficient amperometric systems capable of accurately determining NADH generated by the enzymatic reaction constitutes an essential issue for the development of biosensors suitable to detect target chemical species.

Different electrode coatings have been proposed [1-3], with the aim of solving the problems arising in the direct electrochemical oxidation of NADH at bare electrodes: (i) the high charge transfer overvoltage that shifts the electrochemical process at quite high positive potentials, where the oxidation of interfering species may also take place; and (ii) the massive electrode passivation due to products of the oxidation reaction.

In this framework, it is widely accepted that the $o$-quinone/o-hydroquinone redox couple constitutes an effective redox mediator for NADH oxidation, according to the reactions:

\section{1,2-dihydroxybenzene $\leftrightarrows$ 1,2-benzoquinone $+2 \mathrm{H}^{+}+2 \mathrm{e}^{-}$ \\ 1,2-benzoquinone $+\mathrm{NADH} \leftrightarrows$ 1,2-dihydroxybenzene $+\mathrm{NAD}^{+}$}

Thanks to the occurrence of this electrocatalytic process, NADH oxidation occurs at a notably lower potential value with respect to bare electrode surfaces, i.e., at the potential at which o-hydroquinone is oxidized to $o$-quinone [1-3]. One of the main problems in the realization of efficient amperometric sensors lies in the stability of the anchoring of a similar soluble redox mediator on the electrode surface. Stable chemical bonds can be obtained by using some o-quinone derivatives, mainly consisting of 3,4-dihydroxybenzaldehyde [4], caffeic acid (CFA; see Figure 1) [5,6] or chlorogenic acid [7]. They all undergo reversible charge transfer processes, according to the mechanism described in Figure 1 for the case of CFA.
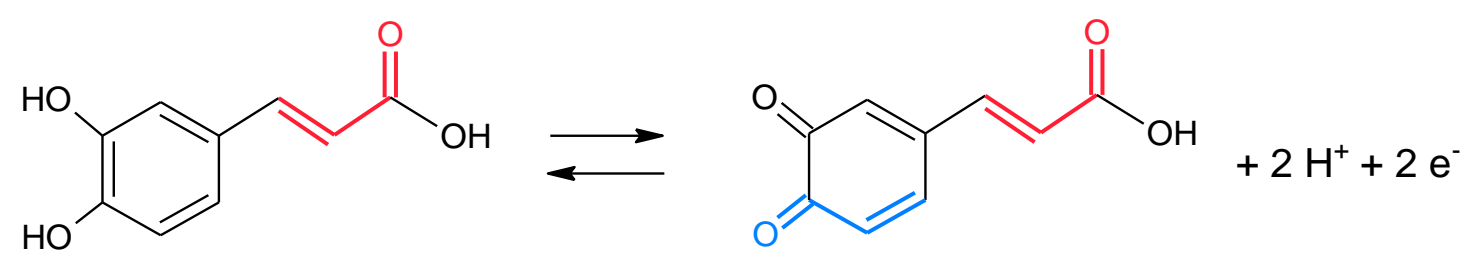

Figure 1. Schematic representation of caffeic acid (CFA) and the relevant oxidized, $o$-quinone form. The colored functional groups represent the potential Michael acceptors exploitable for anchoring the molecule at the carbon-based electrode.

Although the mechanisms through which these species are fixed onto carbon-based surfaces are not univocally accepted, the literature is concordant in concluding that it is based on a Michael addition $[4,5,7]$ performed by carboxyl groups present on the electrode surface, toward the $\beta$ carbon of an $\alpha-\beta$ unsaturated carbonyl moiety (see Figure 1). Aiming at increasing the number of carboxylic groups at the electrode|solution interface, in most cases, the electrode is electrochemically pre-treated by voltammetric scans toward positive potentials [8-10], performed either in acid or basic aqueous solutions. 
Great attention should be paid to the $\mathrm{pH}$ of the deposition solution, in order to address the functionalization of the electrode surface to the expected result [4,5,7]. In particular, the presence of nucleophilic species (e.g., hydroxide ions or deprotonated carboxyl derivatives) in solution may compete with the reactive groups at the electrode surface in the nucleophilic attack to the Michael acceptor. On the other hand, low $\mathrm{pH}$ values partially or completely prevent the reaction, due to the protonation of carboxylic groups on the carbon electrode. As an example, in the case of CFA, the highest coverage is obtained at $\mathrm{pH} 4[5,6]$, i.e., a value slightly lower than $\mathrm{pK}_{\mathrm{a}}(=4.62)$. In this case, however, the literature does not agree in defining whether the nucleophilic attack involves $o$-quinone produced during the electrochemical oxidation (blue-colored in Figure 1) [5] or the Michael acceptor external to the $o$-hydroquinone ring (red-colored in Figure 1) [6].

Among different carbon-based electrodes, nanostructured surfaces should be preferred as the substrate to anchor the redox mediator [11-14]: they attract a great deal of attention in a number of applications, electroanalysis included. One of the most notable advantageous characteristics acknowledged for similar materials lies in the high number of atoms present in correspondence with edge-plane defects. The unsaturated coordination of these atoms renders them particularly efficient in the activation of fast charge transfers occurring via inner-sphere mechanisms, i.e., in promoting electrocatalytic processes. Among the different carbon-based nanosized materials, carbon black nanoparticles (CB) [15-20] show properties very similar to carbon nanotubes, being however characterized by a much lower price, which allows envisaging possible large-scale production of cost-effective devices.

In this paper, we report the development of a screen-printed electrode (SPE) where the surface of the working electrode is modified by a CFA/CB coating, to obtain the CFA/CB/SPE sensor system. The underlying CB layer allows a high number of CFA molecules to be stably deposited, suitable to act as redox mediators for NADH oxidation. The performance of the device at varying the deposition conditions is tested with respect to the electrochemical determination of such an analyte. In particular, CFA was deposited onto an electrochemically-activated carbon surface according to different procedures: (i) electrochemical deposition by cycling the potential within the range where CFA is reversibly oxidized; and (ii) adsorption of the molecule according to a so-called drop-casting method. $\mathrm{CFAel} / \mathrm{CB} / \mathrm{SPE}$ and $\mathrm{CFAad} / \mathrm{CB} / \mathrm{SPE}$ sensor systems were finally obtained, respectively. The advantages of the use of $\mathrm{CFA} / \mathrm{CB} / \mathrm{SPEs}$ are demonstrated in comparison with SPEs modified by a single component, namely CFA/SPE and CB/SPE.

The glycerol dehydrogenase enzyme is finally anchored on the CFA/CB/SPE, in order to preliminary test the applicability of the device as an amperometric biosensor for the determination of glycerol. This chemical species was selected as a typical quality benchmark of grapes [21] and wines [22], in view of potential applications of the investigated biosensor system in food quality control.

\section{Experimental Section}

\subsection{Instruments and Chemicals}

Electrochemical measurements have been carried out with a handheld PalmSens potentiostat/galvanostat (Utrecht, The Netherlands) interfaced with a personal computer. SPEs were 
acquired from EcoBioServices (Florence, Italy) and consisted of a graphite working electrode $(\varnothing=3 \mathrm{~mm})$, an Ag pseudo-reference electrode and a graphite auxiliary electrode.

All reagents were of analytical grade and supplied by Sigma-Aldrich. All solutions used for electrochemical tests were prepared with ultrapure water $(18 \mathrm{M} \Omega \cdot \mathrm{cm}$ resistivity) and contained $0.1 \mathrm{M} \mathrm{KCl}$ supporting electrolyte to stabilize the potential of the reference electrode.

Commercial CB N220 (19-25 nm diameter, $124 \mathrm{~m}^{2} \cdot \mathrm{g}^{-1}$ surface area) was supplied by Cabot Corporation (Ravenna, Italy).

Glycerol dehydrogenase enzyme (GDH, E.C. 1.1.1.6, $112 \mathrm{U} \cdot \mathrm{mg}^{-1}$ ) from Cellulomonas sp. was acquired from Sigma (Milan, Italy) and used without further purification.

\subsection{Preparation and Tests of the Sensor for NADH Detection}

$\mathrm{CB} / \mathrm{SPEs}$ were prepared by modifying the working electrode of SPEs with different aliquots (from 2 to $12 \mu \mathrm{L}$ ) of $1 \mathrm{mg} \cdot \mathrm{mL}^{-1} \mathrm{CB}$ dispersion in $\mathrm{CH}_{3} \mathrm{CN}$, obtained after $1 \mathrm{~h}$ ultrasound sonication.

$\mathrm{CFAel} / \mathrm{SPE}$ and $\mathrm{CFAel} / \mathrm{CB} / \mathrm{SPE}$ were obtained from bare SPE and CB/SPE, respectively, by a two-step electrochemical procedure. In the first step, SPE and CB/SPE were activated in $1 \mathrm{M} \mathrm{H}_{2} \mathrm{SO}_{4}$ aqueous solution, by performing ten subsequent voltammetric cycles between +1.7 to $-1.0 \mathrm{~V}$, at a $0.1 \mathrm{~V} \cdot \mathrm{s}^{-1}$ potential scan rate [23]. The procedure was stopped at $+1.7 \mathrm{~V}$, in order to finally obtain a carbon surface at the highest oxidation degree. CFA was deposited in a subsequent step, by dipping the modified SPEs in a $1 \mathrm{mM}$ CFA, $0.1 \mathrm{M}$ acetate buffer solution ( $\mathrm{pH} 4.0$ ) and by performing ten subsequent potential scans from +0.8 to $-0.1 \mathrm{~V}$ at $0.02 \mathrm{~V} \cdot \mathrm{s}^{-1}$ rate.

Alternatively, CFA was deposited by letting the activated surface of the CB-modified SPE (see above) in contact with a drop of $1 \mathrm{mM} \mathrm{CFA}, 0.1 \mathrm{M}$ acetate buffer solution ( $\mathrm{pH} 4.0$ ), for $1 \mathrm{~h}$, according to the so-called drop-casting method. The electrode surface was then carefully rinsed with abundant distilled water, in order to remove weakly adsorbed molecules. In this case, the device is indicated with the acronym $\mathrm{CFA}_{\mathrm{ads}} / \mathrm{CB} / \mathrm{SPE}$.

In both cases, the effectiveness of CFA deposition was ascertained by performing cyclic voltammetric $(\mathrm{CV})$ experiments in $0.1 \mathrm{M}$ phosphate buffer solution (PB) at pH 7.0. NADH was then added to the same solution, in order to test the electrocatalytic performance of the modified electrode.

The performance of $\mathrm{CFA}_{\mathrm{ads}} / \mathrm{CB} / \mathrm{SPEs}$ with respect to $\mathrm{CFA} / \mathrm{CB} / \mathrm{SPEs}$ has been evaluated by potentiostatic $(E=+0.25 \mathrm{~V})$ amperometric measurements in NADH stirred solutions in the 3-60 $\mu \mathrm{M}$ range of concentrations. Three electrodes realized under the same conditions were tested, and the whole calibration plot was repeated twice on each electrode. The mean slope and the relevant standard deviation were calculated on the basis of the six replicates for each kind of modified SPE. The degree of repeatability of the sensor (D.Repe) has been calculated from the difference between the slope values obtained with the same electrode with respect to the relevant mean value. The value finally reported in Table 1 is the mean between the results obtained for three similar electrodes. The degree of reproducibility (D.Repr) has been calculated from the relative standard deviation of the slope values obtained for three similar electrodes.

\subsection{Preparation and Tests of the Sensor for Glycerol Detection}

For the detection of glycerol, CFAads/CB/SPE was modified by a two-step procedure: In the first step, $1 \%(\mathrm{v} / \mathrm{v})$ glutaraldehyde solution $(2 \mu \mathrm{L})$ was spread onto the surface and allowed to dry for $2 \mathrm{~h}$. 
Afterwards, $1.4 \mu \mathrm{L}$ of PB (pH 8.0), $2.8 \mathrm{UI}$ of GDH and $1.4 \mu \mathrm{L}$ of $1 \%$ (v/v) neutralized Nafion ${ }^{\circledR}$ solution, after carefully mixing, were deposited onto the electrode surface and left to dry overnight.

The sensor was tested by recording CV curves in $0.1 \mathrm{M} \mathrm{PB}(\mathrm{pH} 8.0), 2 \mathrm{mM} \mathrm{NAD}^{+}$, in the absence and in the presence of glycerol at different concentrations, respectively.

\section{Results and Discussion}

The activation of carbon-based surfaces by electrochemical treatment constitutes a procedure widely described in the literature [8-10]. Although a high number of different methods have been proposed, in all cases, the exact nature of the resulting electrode surface remains almost unclear.

In analogy with an effective surface activation procedure previously exploited by us for glassy carbon surfaces [23], SPE and CB/SPEs obtained by depositing different amounts of CB were subjected to ten subsequent voltammetric scans in $1 \mathrm{M} \mathrm{H}_{2} \mathrm{SO}_{4}$ solution. As observed in Figure 2, the voltammetric path shows, in the forward scan, a well-significant current increment at $\mathrm{E}>+1.2 \mathrm{~V}$ and quite a broad reduction peak in the backward scan.

Due to the similarity of these responses with those obtained at Au electrode surfaces [24], it is spontaneous to hypothesize that oxidized functionalities form at the $\mathrm{CB}$ electrode|solution interface during the forward scan, most probably under the form of carboxyls. They are subsequently reduced in the following scan to negative potentials. This assumption is supported by the evidence that the charge spent in the cathodic reduction increases at increasing the amount of $\mathrm{CB}$. This signal, in terms of the corresponding charge spent, can be exploited to give direct evidence of the increase of the apparent electroactive area as a consequence of the deposition of a nanostructured material on the electrode surface [20].

As observed in the inset of Figure 2, the electroactive area increases almost linearly up to the deposition of $6 \mu \mathrm{L}$ of $\mathrm{CB}$, whereas no significant variation is evident for the deposition of higher amounts of nanostructured material. The apparent electroactive area is estimated to increase one order of magnitude with respect to bare SPE. Similar tests performed on three SPEs modified by CB under the same experimental conditions give an indication of the good reproducibility degree of the different CB/SPEs (see the inset of Figure 2).

CFA was deposited on the different CB/SPEs by using either an electrochemical approach or by allowing the solution to be in contact with the activated carbon-based surface. The different CFA/CB/SPEs realized have been tested in pure electrolyte solution, in order to verify the effectiveness of CFA deposition by both deposition methods. In all cases, the voltammetric curve recorded shows a well-defined anodic-cathodic peak system, typical of the quinone-hydroquinone redox couple (see the blue curve in Figure 3A). As expected [4,7], the relevant peak currents progressively decrease during the first potential cycles and at a much lower rate in the following scans. The current peak values result in being directly proportional to the potential scan rate within the range $0.02-0.50 \mathrm{~V} \cdot \mathrm{s}^{-1}$, indicating the occurrence of a surface-confined electrochemical process.

$\mathrm{CFA} / \mathrm{CB} / \mathrm{SPEs}$ were tested with respect to the determination of $\mathrm{NADH}$. As observed in the exemplificative voltammograms reported in Figure 3A, the peak due to oxidation of the CFA redox mediator increases at increasing concentration of NADH in solution, indicating the active role played by this chemical species in the electrocatalytic oxidation of the analyte. Qualitatively similar results 
were obtained using different CFA/CB/SPEs, independently of the amount of the deposited CB and of the procedure followed for CFA deposition.

A deeper investigation concerning the effect of the amount of $\mathrm{CB}$ deposited on the response of the sensor system was carried out with $\mathrm{CFAel} / \mathrm{CB} / \mathrm{SPEs}$. As observed by comparing the responses obtained in $1 \mathrm{mM} \mathrm{NADH}$, subtracted by the relevant background signal, the sensitivity of the sensor response increases by increasing the volume of CB dispersion deposited on SPEs up to $6 \mu \mathrm{L}$ (see Figure 3B). For this reason, SPEs modified with this amount of CB were chosen for further investigations.

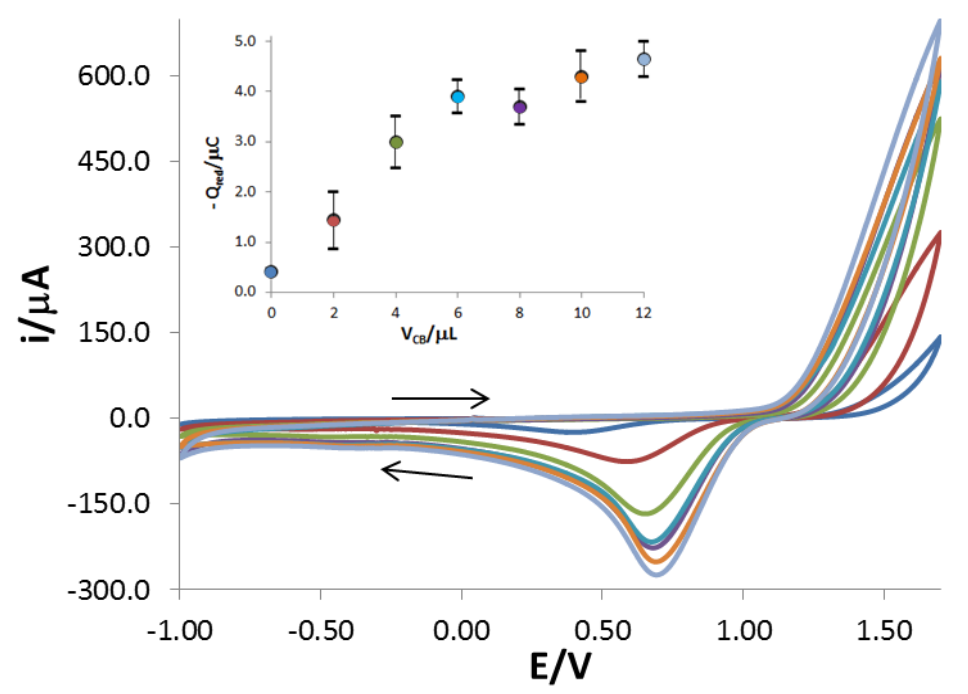

Figure 2. Tenth voltammetric scan obtained at the SPE and at different CB/SPEs in $1 \mathrm{M}$ $\mathrm{H}_{2} \mathrm{SO}_{4}, 0.1 \mathrm{M} \mathrm{KCl}$ solution by varying the amount of $\mathrm{CB}$ deposited on the electrode surface; $0.05 \mathrm{~V} / \mathrm{s}$ potential scan rate. The relevant trend of the charge spent in the reduction peak $v s$. the volume of CB dispersion deposited is reported in the inset. The error bar length accounts for twice the standard deviation of four replicates for each deposition condition.
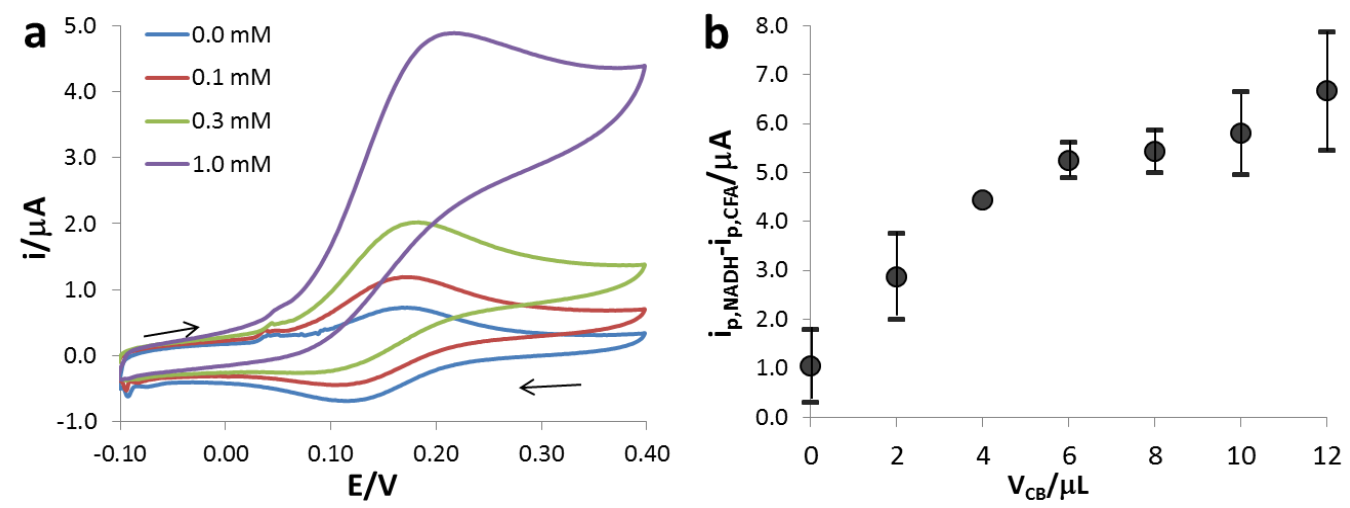

Figure 3. (a) Typical CV responses of CFA/CB/SPEs in $0.1 \mathrm{M} \mathrm{PB}(\mathrm{pH} 7.0), 0.1 \mathrm{M} \mathrm{KCl}$ in the absence (blue line) and in the presence of different NADH concentrations: $0.1 \mathrm{mM}$ (red line), $0.3 \mathrm{mM}$ (green line) and $1.0 \mathrm{mM}$ (purple line); $0.02 \mathrm{~V} \cdot \mathrm{s}^{-1}$ potential scan rate.

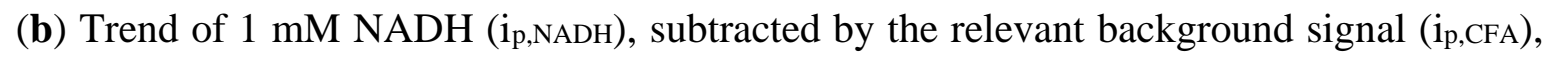
vs. the volume of $\mathrm{CB}$ dispersion deposited on $\mathrm{CFAel} / \mathrm{CB} / \mathrm{SPEs}$. The error bar length accounts for two-times the standard deviation, computed by considering results coming from three similar $\mathrm{CFA}_{\mathrm{el}} / \mathrm{CB} / \mathrm{SPEs}$. 
The effectiveness of $\mathrm{CFA} / \mathrm{CB}$ coating in promoting the electrocatalytic activity toward NADH oxidation was also proven by comparing the responses obtained with those on SPEs modified with a single component of the coating, i.e., either CFA or CB. As observed in Figure 4, NADH oxidation is significantly anticipated for $\mathrm{CFA} / \mathrm{SPE}$ and $\mathrm{CFA} / \mathrm{CB} / \mathrm{SPE}$, with respect to $\mathrm{CB} / \mathrm{SPE}$. Concurrently, significantly higher current peaks can be obtained when CFA is anchored on an underlying CB coating, with respect to CFA/SPEs, in which the redox mediator is directly deposited on bare carbon electrodes.

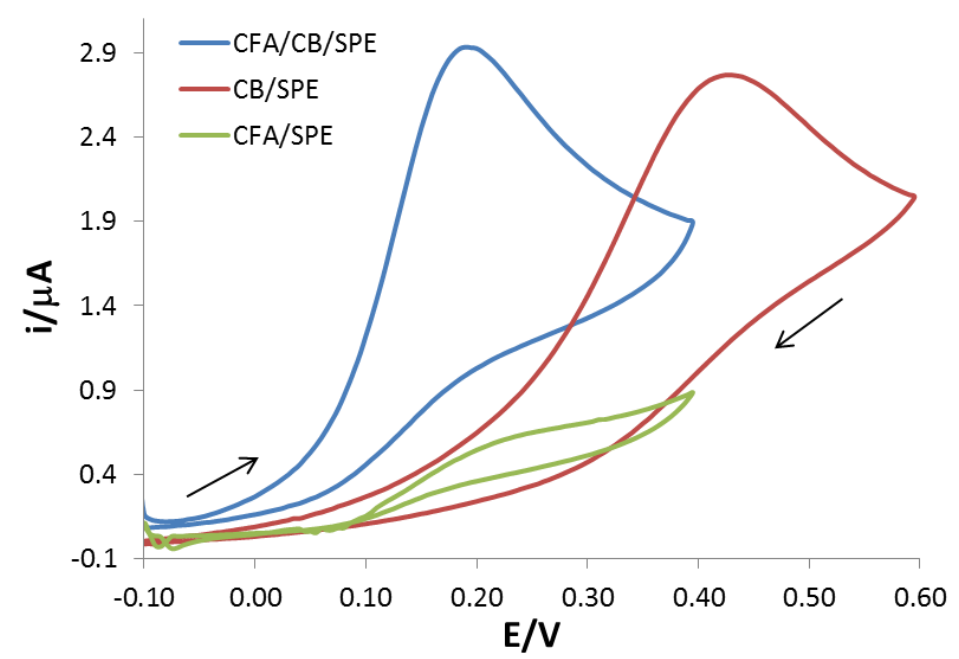

Figure 4. $\mathrm{CV}$ responses of $0.5 \mathrm{mM} \mathrm{NADH}$, subtracted by the relevant blank signal, collected in $0.1 \mathrm{M} \mathrm{PB}(\mathrm{pH} 7.0), 0.1 \mathrm{M} \mathrm{KCl}$ at the different modified SPEs.

It is, thus, possible to conclude that the use of CFA/CB/SPEs allows the application of particularly low potential values for potentiostatic amperometric determination of $\mathrm{NADH}$, namely $+0.25 \mathrm{~V}$. On the contrary, the application of a potential value as high as $+0.50 \mathrm{~V}$ is necessary in the case of CB/SPE, in order to reach the constant current values. This aspect is of utmost importance in the case of amperometric biosensors, because the interference of additional species present in the solution can be avoided.

Repeated tests for NADH determination have been performed with CFA/CB/SPEs, in order to evidence possible differences in the sensor performance due to the dissimilar procedure used for CFA deposition. In this respect, it has to be evidenced that the electrochemical deposition is the procedure generally reported by the literature [5-7,25]. However, by considering that the mechanisms proposed for CFA deposition on carbon surfaces are based on Michael addition, the drop-casting method represents an alternative deposition approach, which gives the $\mathrm{CFA}_{\mathrm{ads}} / \mathrm{CB} / \mathrm{SPE}$ sensor system. [26]. In principle, only the Michael acceptor external to the $o$-hydroquinone ring (red-colored in Figure 1) should be involved in the covalent bond with the oxidized moieties on the carbon surface. On the contrary, electrochemical deposition may also involve direct attack of the phenyl ring (blue-colored in Figure 1), once $o$-quinone is produced from the electrochemical oxidation of CFA.

The performance of the two sensor systems has been defined by amperometric tests carried out at a fixed applied potential of $+0.25 \mathrm{~V}$. The relevant parameters defining the effectiveness of the sensing systems are reported in Table 1. 
Table 1. Performance of CFA/carbon black (CB)/screen-printed electrodes (SPEs) toward $\mathrm{NADH}$ determination calculated from amperometric measurements at $\mathrm{a}+0.25 \mathrm{~V}$ constant potential; the relevant standard deviation, calculated by considering three similar SPEs, is reported within brackets.

\begin{tabular}{ccc}
\hline & $\mathbf{C F A}_{\mathrm{e}} / \mathbf{C B} / \mathbf{S P E}$ & $\mathbf{C F A}_{\text {add }} / \mathbf{C B} / \mathbf{S P E}$ \\
\hline $\mathbf{i}_{\mathrm{p}, \text { CFA }}(\boldsymbol{\mu} \mathbf{A}) *$ & $0.85(0.26)$ & $0.49(0.16)$ \\
sensitivity $\left(\boldsymbol{\mu A} \cdot \boldsymbol{\mu} \mathbf{M}_{\mathbf{N A D H}}^{-\mathbf{1}}\right)$ & $0.007(0.003)$ & $0.012(0.001)$ \\
$\mathbf{L O D}(\boldsymbol{\mu M})$ & 3.7 & 1.6 \\
D.Repe (\%) & 95.6 & 96.6 \\
D.Repr (\%) & 56.4 & 89.7 \\
\hline
\end{tabular}

* This value is calculated from voltammetric traces collected in pure electrolyte solution at a $0.02 \mathrm{~V} \cdot \mathrm{s}^{-1}$ potential scan rate.

The values of the current peak due to CFA oxidation in PB solution ( $\left.\mathrm{i}_{\mathrm{p}, \mathrm{CFA}}\right)$ indicate that the amount of redox mediator fixed on the substrate is higher when using the electrochemical method. On the contrary, the sensitivity for NADH detection is surprisingly higher in the case of $\mathrm{CFA}_{\text {ads }} / \mathrm{CB} / \mathrm{SPE}$. This fact suggests that better sensor performance, in terms of sensitivity, is obtained when the anchoring method leaves the catechol functionality unreacted.

It is also worth evidencing that the higher sensitivity observed in the case of $\mathrm{CFA}_{\mathrm{ads}} / \mathrm{CB} / \mathrm{SPE}$ is associated with lower noise in the signal registered, i.e., the values of the standard deviation of both the slope and intercept of the calibration line are lower in this case. This fact positively affects the value of the relevant limit of detection (LOD), calculated as the quantity corresponding to a signal obtained by the intercept value plus three-times the relevant standard deviation. Finally, it should be noticed that this simple deposition method combines the high repeatability degree, observed for any kind of $\mathrm{CFA} / \mathrm{CB}$ deposit, with quite a high reproducibility degree, which constitutes a drawback in the case of $\mathrm{CFAel} / \mathrm{CB} / \mathrm{SPE}$ systems. This last aspect constitutes quite a critical point in the case of disposable devices.

Thanks to these encouraging results, the $\mathrm{CFA}_{\mathrm{ads}} / \mathrm{CB} / \mathrm{SPE}$ system was tested as a platform for the deposition of GDH, in order to envisage a sensor for the detection of glycerol, namely $\mathrm{GDH} / \mathrm{CFA}_{\mathrm{ads}} / \mathrm{CB} / \mathrm{SPE}$. The stable anchoring of the enzyme at the electrode-solution interface was achieved by exploiting one of the most diffused approaches, namely cross-linking with glutaraldehyde. Due to the high activity of this enzyme in slightly alkaline media [27], the measurements were carried out in $\mathrm{PB}$ at $\mathrm{pH}=8.0$.

As expected, the current peak due to CFA oxidation shifts to less positive potentials with respect to that recorded in voltammetric tests at $\mathrm{pH}$ 7.0. This implies that constant current values for NADH oxidation in amperometric tests can be reached at potential values as low as $+0.20 \mathrm{~V}$. As observed in Figure 5, the addition of glycerol in solution leads to a current increment in the correspondence of CFA oxidation. In these explorative tests, an increase of the voltammetric peak current at increasing concentration is verified. This behavior evidences that the GDH/CFAads/CB/SPE sensor system can be reasonably thought to constitute an efficient system for the quantification of such an analyte. 


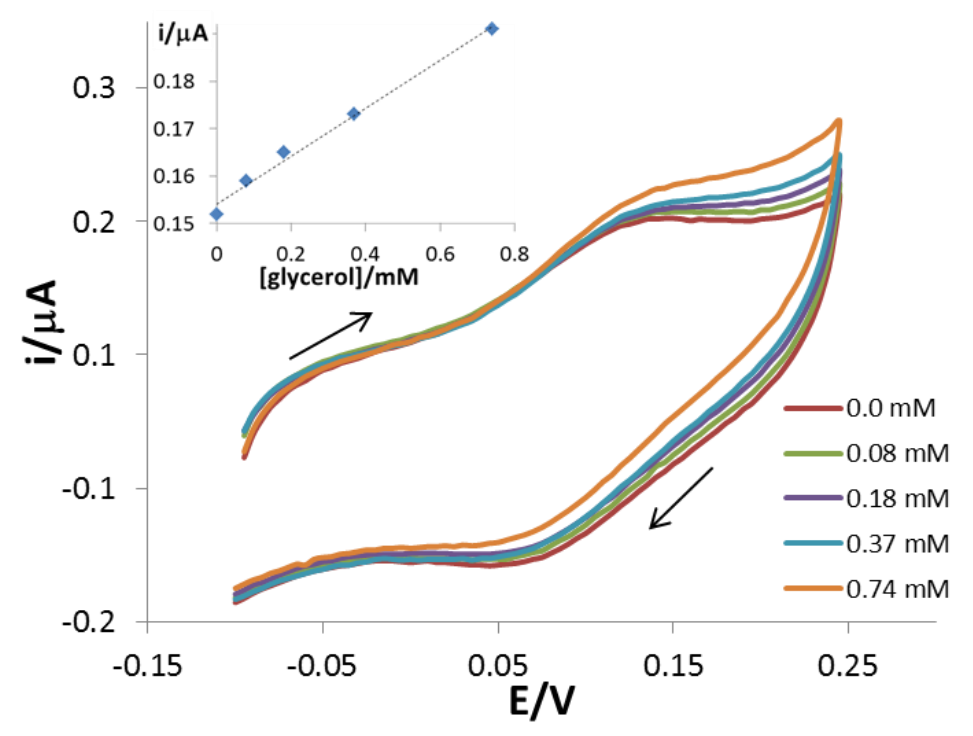

Figure 5. $\mathrm{CV}$ responses of glycerol dehydrogenase enzyme $(\mathrm{GDH}) / \mathrm{CFA}_{\mathrm{ads}} / \mathrm{CB} / \mathrm{SPEs}$ in $0.1 \mathrm{M} \mathrm{PB}(\mathrm{pH} 8.0), 0.1 \mathrm{KCl}, 2 \mathrm{mM} \mathrm{NAD}^{+}$in the absence and in the presence of glycerol at different concentrations; $0.005 \mathrm{~V} \cdot \mathrm{s}^{-1}$ potential scan rate; the inset reports the plot of the current values registered at $+0.20 \mathrm{~V}$ with respect to the relevant glycerol concentration.

\section{Conclusions}

A new disposable sensor, consisting of a CFA/CB-modified SPE, has been developed and finalized to the determination of $\mathrm{NADH}$, a species of outstanding importance in the framework of enzymatic biosensing. The presence of CFA induces effective electrocatalytic activity with respect to the oxidation of this species, finally resulting in a significant anticipation of the electrochemical response. At the same time, the use of a carbon nanostructured surface allows a high number of CFA molecules to be stably anchored on the electrode, which strongly enhances the sensor sensitivity in comparison with similar systems that only contain CFA molecules.

Further modification of CFA/CB/SPE with glycerol dehydrogenase enzyme leads to a sensor system for glycerol detection. The first tests in this direction encourage deepening the potentialities of this biosensor in view of the potential applications in food quality control.

\section{Acknowledgments}

The authors kindly acknowledge the Ateneo of Modena and Reggio Emilia for the financial support in the framework of Linee Strategiche di Ateneo: "Nano- and emerging materials and systems for sustainable technologies".

\section{Author Contributions}

All authors contributed to the final work, capitalizing their own experience for the realization of the final device. In particular, Chiara Zanardi exploited expertise in the development of nanostructured surfaces for sensors and biosensors realization; Erika Ferrari that in the reactivity of molecules possessing $\alpha-\beta$ unsaturated carbonyl moieties; Laura Pigani in the electrochemical behavior of 
polyphenols; Fabiana Arduini in the use of carbon black in electroanalytical applications; and Renato Seeber in the supervision of the whole research activity.

\section{Conflicts of Interest}

The authors declare no conflict of interest.

\section{References}

1. Gorton, L.; Domínguez. E. Electrocatalytic oxidation of $\mathrm{NAD}(\mathrm{P}) \mathrm{H}$ at mediator-modified electrodes. Rev. Mol. Biotechnol. 2002, 82, 371-392.

2. Prieto-Simón, B.; Fàbregas, E. Comparative study of electron mediators used in the electrochemical oxidation of NADH. Biosens. Bioelectron. 2004, 19, 1131-1138.

3. Radoi, A.; Compagnone, D.; Recent advances in NADH electrochemical sensing design. Bioelectrochemistry 2009, 76, 126-134.

4. Pariente, F.; Tobalina, F.; Darder, M.; Lorenzo, E.; Abruña, H.D. Electrodeposition of redox-active films of dihydroxybenzaldehydes and related analogs and their electrocatalytic activity toward NADH oxidation. Anal. Chem. 1996, 68, 3135-3142.

5. Zare, H.R.; Golabi, S.M. Caffeic acid modified glassy carbon electrode for electrocatalytic oxidation of reduced nicotinamide adenine dinucleotide (NADH). J. Solid State Electrochem. 2000, 4, 87-94.

6. Lee, P.T.; Compton, R.G. Electrochemical detection of NADH, cysteine, or glutathione using a caffeic acid modified glassy carbon electrode. Electroanalysis 2013, 25, 1613-1620.

7. Zare, H.R.; Golabi, S.M. Electrocatalytic oxidation of reduced nicotinamide adenine dinucleotide $(\mathrm{NADH})$ at a chlorogenic acid modified glassy carbon electrode. J. Electroanal. Chem. 1999, 464, 14-23.

8. McCreery, R.L. Carbon electrodes: Structural effects on electron transfer kinetics. In Electroanalytical Chemistry; Bard, A.J., Ed.; Marcel Dekker: New York, NY, USA, 1991; Volume 17, pp.221-374.

9. Bowers, M.L.; Yenser, B.A. Electrochemical behavior of glassy carbon electrodes modified by electrochemical oxidation. Anal. Chim. Acta 1991, 243, 43-53.

10. Dekanski, A.; Stevanović, J.; Stevanović, R.; Nikolić, B.Ž.; Jovanovic, V.M. Glassy carbon electrodes. I. Characterization and electrochemical activation. Carbon 2001, 39, 1195-1205.

11. Seeber, R.; Terzi, F.; Zanardi, C. Nanosized materials. In Functional Materials in Amperometric Sensing; Scholz, F., Ed.; Springer-Verlag: Heidelberg, Germany, 2014; pp. 139-182.

12. Agüí, L.; Yáñez-Sedeño, P.; Pingarrón, J.M. Role of carbon nanotubes in electroanalytical chemistry: A review. Anal. Chim. Acta 2008, 622, 11-47.

13. Shao, Y.; Wang, J.; Wu, H.; Liu, J.; Aksay, I.A.; Lin, Y. Graphene based electrochemical sensors and biosensors: A review. Electroanalysis 2010, 22, 1027-1036.

14. Marín, S.; Merkoçi, A. Nanomaterials based electrochemical sensing applications for safety and security. Electroanalysis 2012, 24, 459-469. 
15. Arduini, F.; Amine, A.; Majorani, C.; Di Giorgio, F.; de Felicis, D.; Cataldo, F.; Moscone, D.; Palleschi, G. High performance electrochemical sensor based on modified screen-printed electrodes with cost-effective dispersion of nanostructured carbon black. Electrochem. Comm. 2010, 12, 346-350.

16. Arduini, F.; Di Nardo, F.; Amine, A.; Micheli, L.; Palleschi, G.; Moscone, D. Carbon black-modified screen-printed electrodes as electroanalytical tools. Electroanalysis 2012, 24, 743-751.

17. Carvalho, R.C.; Mandil, A.; Prathish, K.P.; Amine, A.; Brett, C. Carbon nanotube, carbon black and copper nanoparticle modified screen printed electrodes for amino acid determination. Electroanalysis 2013, 25, 903-913.

18. Hou, C.; Tang, W.; Zhang, C.; Wang, Y.; Zhu, N. A novel and sensitive electrochemical sensor for bisphenol. A determination based on carbon black supporting ferroferric oxide nanoparticles. Electrochim. Acta 2014, 144, 324-331.

19. Vicentini, F.C.; Ravanini, A.E.; Figueiredo-Filho, L.C.S.; Iniesta, J.; Banks, C.E.; Fatibello-Filho, O. Imparting improvements in electrochemical sensors: Evaluation of different carbon blacks that give rise to significant improvement in the performance of electroanalytical sensing platforms. Electrochim. Acta 2015, 157, 125-133.

20. Arduini, F.; Zanardi, C.; Cinti, S.; Terzi, F.; Moscone, D.; Palleschi, G.; Seeber, R. Effective electrochemical sensor based on screen-printed electrodes modified with a carbon black-Au nanoparticles composite. Sens. Actuators B Chem. 2015, doi:10.1016/j.snb.2015.02.051.

21. Zanardi, C.; Ferrari, L.; Zanfrognini, B.; Pigani, L.; Terzi, F.; Cattini, S.; Rovati, L.; Seeber, R. Development of a sensor system for the determination of sanitary quality of grapes. Sensors $\mathbf{2 0 1 3}$, 13, 4571-4580.

22. Gamella, M.; Campuzano, S.; Reviejo, A.J.; Pingarron J.M. Integrated multienzyme electrochemical biosensors for the determination of glycerol in wines. Anal. Chim. Acta 2008, 609, 201-209.

23. Terzi, F.; Palacios Santander, J.M.; Zanardi, C.; Pigani, L.; Naranjo-Rodríguez, I.; Hidalgo-Hidalgo de Cisneros, J.L.; Seeber, R. Electrocatalytic and antifouling properties of glassy carbon/metal oxide electrode systems. J. Solid State Electrochem. submitted.

24. Sustersic, M.G.; Almeida, N.V.; von Mengershausen, A.E.; Esquenoni, S.M. Hydrogen oxidation on gold electrode in sulphuric acid solution. Int. J. Hydrog. Energy 2012, 37, 14747-14752.

25. Lee, P.T.; Ward, K.R.; Tschulik, K.; Chapman, G.; Compton, R.G. Electrochemical detection of glutathione using a poly(caffeic acid) nanocarbon composite modified electrode. Electroanalysis 2014, 26, 366-373.

26. Salami, A.; Miranzadeh, L.; Hallaj, R. Amperometric and voltammetric detection of hydrazine using glassy carbon electrodes modified with carbon nanotubes and catechol derivatives. Talanta 2008, 75, 147-156.

27. Eftekhari, A. Glycerol biosensor based on glycerol dehydrogenase incorporated into polyaniline modified aluminum electrode using hexacyanoferrate as mediator. Sens. Actuators B Chem. 2001, 80, 283-289.

(C) 2015 by the authors; licensee MDPI, Basel, Switzerland. This article is an open access article distributed under the terms and conditions of the Creative Commons Attribution license (http://creativecommons.org/licenses/by/4.0/). 